\title{
BMJ Open Quality Preoperative fasting in the department of plastic surgery
}

\author{
Andrew Davies, ${ }^{1}$ Wei Sheng Pang, ${ }^{2}$ Timothy Fowler, ${ }^{3}$ Ffion Dewi, ${ }^{3}$ Thomas Wright ${ }^{3}$
}

To cite: Davies A, Pang WS, Fowler T, et al. Preoperative fasting in the department of plastic surgery. BMJ Open Quality 2018;7:e000161. doi:10.1136/

bmjoq-2017-000161

- Additional material is published online only. To view please visit the journal online (http://dx.doi.org/10.1136/ bmjoq-2017-000161).

Received 9 July 2017 Revised 21 July 2018 Accepted 21 August 2018

Check for updates

(C) Author(s) (or their employer(s)) 2018. Re-use permitted under CC BY-NC. No commercial re-use. See rights and permissions. Published by BMJ.

${ }^{1}$ Department of Orthopaedics, North Bristol NHS Trust, Bristol, UK

${ }^{2}$ University of Bristol Medical School, Bristol, UK

${ }^{3}$ Department of Plastic Surgery, North Bristol NHS Trust, Bristol, UK

Correspondence to

Andrew Davies;

ardavies@doctors.org.uk

\section{ABSTRACT}

Preoperative fasting is necessary to reduce the risk of regurgitation of gastric contents and pulmonary aspiration in patients undergoing general anaesthetic and procedural sedation. Excessive fasting is associated with metabolic, cardiovascular and gastrointestinal complications and patient discomfort. We aimed to reduce the fasting time for patients on the plastic surgery trauma list. Adult inpatients awaiting surgery were asked to complete a preoperative assessment sheet. Questions included the length of preoperative fasting, clarity of instructions and wellness scores. Three cycles of data collection were performed over a 12-month period, patients who declined to participate or were unable to consent were excluded. The first cycle revealed the need for significant improvement. Interventions included staff education, patient information sheets, preoperative drinks, greater availability of ward snacks and improved communication between the ward staff and surgical team through our electronic trauma database. The initial audit of 15 patients revealed a mean fasting time of 16.3 hours for fluid (range 10-22) and a mean of 19.3 hours for solid food (range 10-24). The mean wellness score was $6 / 10$ (10 being very well), $67 \%$ of patients felt they were given clear information. The final cycle demonstrated clear improvement in all domains. The mean fasting time declined to 5.1 hours for fluid (range 3-10 hours) and 13 hours for solid food (range 7.5-17 hours). The mean wellness score ( $10=$ very well) increased from 6 to 8 , the mean thirst score declined from 6.1 to 5.1 and $100 \%$ patients felt they had been given clear information. Removal of the traditional 'NBM from midnight', patient education, a clear fasting routine with preoperative drinks and improved communication between the full multidisciplinary team has led to a reduction in the fasting times on our trauma list.

\section{PROBLEM}

The plastic surgery department at our Trust provides a daily trauma list for adult patients referred from the local and wider area. Approximately 1700 trauma cases are performed per year. Patients are admitted through the emergency department or trauma clinic, for surgery at the next available opportunity. This includes patients requiring complex softtissue reconstruction following major trauma. Nursing and medical staff noted that inpatients awaiting surgery were often fasting for excessive periods. Several issues contribute to this. Previous dogma has resulted in a 'nil by mouth from midnight' policy. Due to the unpredictable nature of the trauma lists in this major trauma centre, patients may be operated on later than expected and communication between the theatre and ward staff is challenging during busy shifts. Patients may not be given consistent advice regarding eating and drinking times and ward staff felt communication with theatre teams could be improved. Additionally, the availability of meals and snacks during the night became an area of greater focus. Our goal was to reduce the fasting time for inpatients on the plastic surgery trauma list by $50 \%$ over a 12 -month period. This project was supported by the full multidisciplinary team (MDT) and the efficacy of our interventions was tested through several cycles of data collection.

\section{BACKGROUND}

Preoperative fasting is recommended to prevent pulmonary aspiration of gastric contents, which is associated with significant morbidity and mortality. The practice of preoperative fasting has evolved over the 20th century. Work published by Mendelson highlighted the risk of pulmonary aspiration in obstetric patients receiving nitrous oxide and ether anaesthetic. ${ }^{1}$ Traditionally a complete fast of at least 5 hours was recommended and many advocated 'nil by mouth from midnight'; gastric emptying studies have since demonstrated this is unnecessary. ${ }^{2-4}$ Surgery places significant stress on the body. Insulin resistance increases both during surgery and in the fasting state. ${ }^{5}$ Surgery and other trauma also increases our metabolic rate, resulting in accelerated catabolism. Minimising the preoperative fast reduces postoperative insulin resistance and the surgical stress response leading to improved postoperative recovery.

Recent international consensus guidelines (2017) recommend a minimum fasting period of 6 hours for food and 2 hours for clear fluid. ${ }^{6}$ Clear fluids include water, fruit juices without pulp, clear tea and black coffee. 


\section{BASELINE MEASUREMENT}

Outcome measurements included; the total fasting time for food and fluid, patients' preoperative thirst and 'wellness' scores and the clarity of information given to patients. These were assessed through a preoperative patient questionnaire (document 1) completed with patients on the morning of surgery, as close to the time of induction as possible. Anaesthetic charts and patient notes were reviewed to determine the exact timing of surgery and the provision of intravenous fluid in the preoperative period. Measurements were selected to assess both the fasting time and the influence this had on the patient experience.

The preoperative questionnaire included five simple questions:

1. What time did you last consume any clear fluid?

2. What time did you last consume food?

3. Statement: I have received clear information about whether I am allowed to eat and drink prior to this operation (disagree, neutral, agree).

4. How thirsty do you feel? (scale $1-10,10=$ very thirsty).

5 . How well do you feel? (scale $1-10,10=$ verywell).

This study included all consenting adult patients admitted overnight for surgery on the plastics trauma list during our study periods. Our baseline measurements from 15 patients were completed during July 2016 and revealed excessive periods of fasting prior to surgery. The mean fasting time was 19.3 hours for solid food and 16.3 hours for clear fluid. Six patients received intravenous fluids during the preoperative period. Sixty-seven per cent agreed they had been given clear information about preoperative fasting, however, the content of the recommendations they were given was unclear. Patients reported a mean score of 6.1 for thirst $(10=$ very thirsty) and a mean 'wellness' score of 6 ( $10=$ verywell $)$.

See online supplementary file 1.

\section{DESIGN}

A meeting was held with the ward sister and senior nurses to determine the key challenges. This initially highlighted two main areas; staff education and improved communication with the ward regarding the timing of surgery. Frequently, members of the surgical team requested patients were 'nil by mouth from midnight'. This would be applied in all cases if there was a chance patients were having surgery the following day. Surgery may not be performed until the afternoon, which was often predictable following the morning trauma meeting.

Patients receiving surgery on an outpatient basis were routinely phoned by our trauma coordinators to inform them about the timing of surgery and give advice regarding eating and drinking. For inpatients we did not have a robust system of patient education. We searched through current trust information sheets and felt a new, simple laminated patient information sheet would best inform patients of the necessary information.
Following our first complete audit cycle, we planned to present the work at our departmental audit meeting, to gather feedback and incorporate further recommendations from the MDT.

\section{STRATEGY}

Plan-Do-Study-Act (PDSA) cycle 1: Improved communication. Education of staff and patients.

Discussions were held at several nurse handovers to educate staff regarding replacement of 'nil by mouth from midnight' with 'food until 02:00 hours, fluid until 06:00 hours'. All junior doctors were contacted to update them on the Trust fasting policy and ensure preoperative management plans were consistent. The ward sister and senior staff nurses were provided with access to the department's 'shared' intranet folder. This contained the daily trauma list with the expected patient order, which is updated during the morning trauma meeting. Induction packs for junior doctors were updated to ensure each new cohort are fully aware of the Trust fasting policy and the processes in place to reduce fasting times (document 2).

During the process of an acute admission, patients were not always clear about when they could eat and drink, and the benefits of doing so. We designed a short information sheet with key information about preoperative fasting, and encouraged patients to eat and drink up until the specified times.

PDSA cycle 2: preoperative drinks and snack boxes.

The analysis of our results following the second cycle of data collection demonstrated that despite improvement, patients were frequently only eating and drinking the evening before surgery. These challenges were discussed at our departmental audit meeting. Preoperative carbohydrate drinks were being used successfully in other surgical departments. After discussion with our pharmacy department, these were provided on the plastic surgery ward, and the catering team ensured sandwiches and snacks were available at all times. Preoperative drinks are routinely prescribed on the morning of surgery at 06:00 hours.

See online supplementary file 2 .

\section{RESULTS}

Our initial interventions led to improved fasting times. The mean fasting time decreased to 14.8 hours for food (range 8.5-23.5 hours) and 9.5 hours for clear fluid (range 3-23.5 hours). Seventy-three per cent of patients felt they were given clear information. However, continued improvement was necessary and the morning preoperative routine was highlighted as an area for focused intervention. The results of our final cycle of 15 patients revealed a further decline in the fasting time to 13 hours for food (range 7.5-17hours) and 5.1 hours for fluid (range $3-10$ hours), $31 \%$ of the initial fluid fasting time (figure 1). When patients were asked how well they felt on a scale of 1-10 (10 being very well), the mean score increased to 8 (figure 2). The mean score for thirst (10 


\section{Mean fasting time}

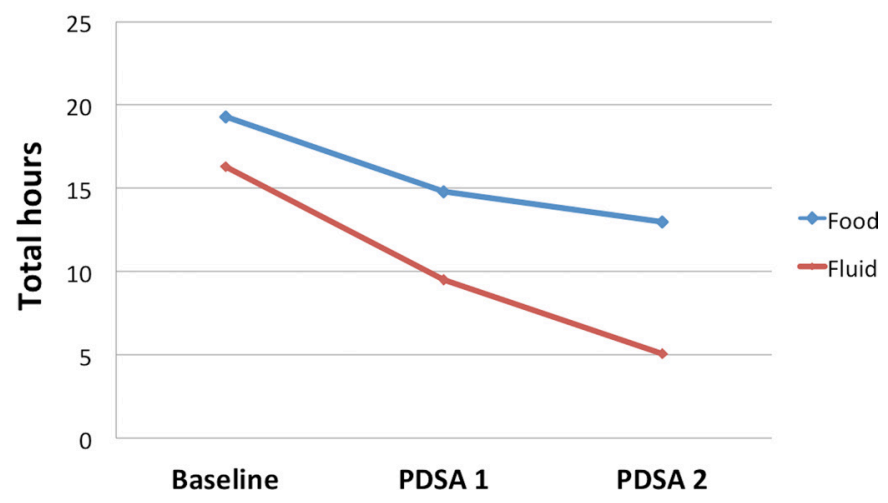

Figure 1 Run chart fasting time.

being very thirsty) reduced to 5.1. One hundred per cent of patients agreed they had been given clear information.

See online supplementary files-'figures 1 and 2'.

\section{LESSONS AND LIMITATIONS}

Despite long initial mean fasting times, this project highlighted how shared purpose and clear goals within a MDT, can lead to significant improvements in patient care. There has been a clear shift in preoperative fasting practice in our department. 'Nil by mouth from midnight' is no longer practised and preoperative drinks have become routine. Prompt and reliable communication between doctors, nurses, patients and theatre staff was vital to ensuring patients were adequately prepared for surgery without unnecessary starvation. Throughout this study no procedures were delayed due to inadequate fasting times.

We considered the sustainability of our interventions central to the success of this project. Clinical staff are under constant pressure from a variety of sources. To avoid increasing or duplicating work; database access allowed senior nurses to clarify the list order at their convenience, this information could then be disseminated to the ward team as required. Patient information booklets are used with good effect in our outpatient clinics. On the acute trauma ward, these may not be available at all times, and patients may not have the opportunity to read through a

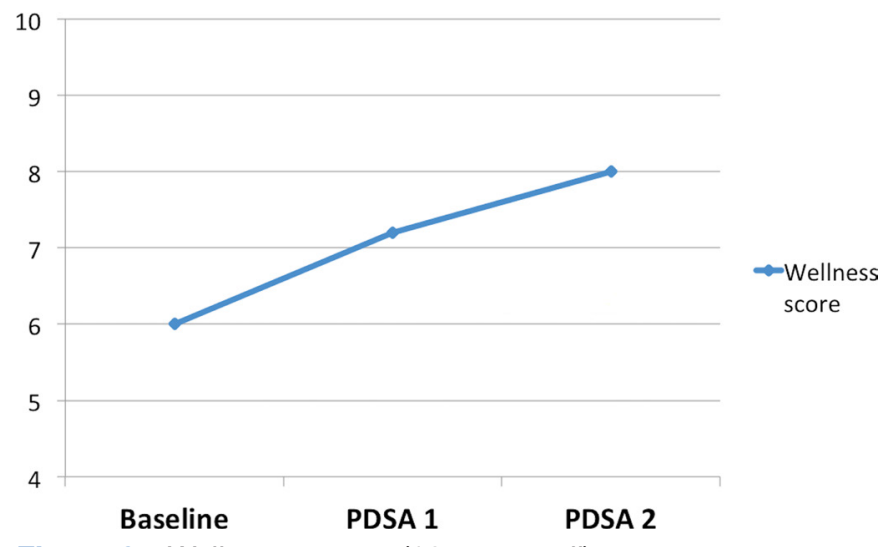

Figure 2 Wellness scores (10=very well). comprehensive booklet. Our simple and clear laminated sheets provide a readily available resource that highlights this key issue. These sheets also serve as a prompt for student nurses, healthcare assistants and catering staff that may have recently rotated onto the ward and may not be familiar with this practice. Furthermore the new information included in the junior doctors' induction packs ensures each new cohort adheres to the updated fasting policy and is aware of the patient information sheets, carbohydrate drinks and catering support available.

The introduction of pre-operative carbohydrate drinks at 06:00 hours has formalised the process of eating and drinking on the morning of surgery and prevents ambiguity. Nursing staff are now confident these can be given pre-operatively in the majority of cases, and they act as a prompt to patients each morning.

In addition to achieving the full benefit of our current strategies, we acknowledge that ongoing re-evaluation, with additional interventions is required to ensure continued improvement. In particular, going forward we will focus on those patients who receive their operations later in the day. Trauma lists are frequently busy, we do not wish to delay procedures unnecessarily because patients are not starved. However, when lists are full, patients will receive a morning snack if their procedure is planned for 15:00 hours or later. Continuous re-evaluation of this strategy will be required to prevent any delay to surgery.

\section{CONCLUSION}

The evidence for preoperative fasting has evolved significantly over the last century. Clinical practice has lagged behind recommendations and the tradition of fasting overnight continues to be employed, leading to the excessive fasting times recognised in our department. Our strategy for change was developed in consultation with all members of the MDT and evolved following presentation at trust audit meetings during the PDSA cycles. Improved communication within the MDT, staff and patient education, modification to our prescribing practice and an enhanced catering service has led to a significant improvement in fasting times and the perioperative experience of our patients. We were careful to ensure our interventions did not increase staff work load on the busy trauma ward, consequently we feel this quality improvement work will lead to sustained improvement.

Acknowledgements Thanks to Simimole George.

Contributors AD: Project design, data collection and analysis, implementation of interventions, writing and review of the manuscript. WSP: Data collection and analysis, writing and review of the manuscript. TF and FD: Data collection and analysis, implementation of interventions, manuscript review. TW: Project supervisor, implementation of interventions, manuscript review.

Funding The authors have not declared a specific grant for this research from any funding agency in the public, commercial or not-for-profit sectors.

Competing interests None declared.

Ethics approval The study was registered with our Trust audit and research department. Formal ethical approval was not sought for this service improvement work. 
Provenance and peer review Not commissioned; externally peer reviewed.

Open access This is an open access article distributed in accordance with the Creative Commons Attribution Non Commercial (CC BY-NC 4.0) license, which permits others to distribute, remix, adapt, build upon this work non-commercially, and license their derivative works on different terms, provided the original work is properly cited, appropriate credit is given, any changes made indicated, and the use is non-commercial. See: http://creativecommons.org/licenses/by-nc/4.0/.

\section{REFERENCES}

1. Mendelson CL. The aspiration of stomach contents into the lungs during obstetric anaesthesia. Am J Obstet Gynaecol 1946;52:191-205.

2. Maltby JR, Sutherland AD, Sale JP, et al. Preoperative oral fluids: is a five-hour fast justified prior to elective surgery? Anesth Analg 1986;65:1112-6.
3. Levy DM. Pre-operative fasting -60 years on from Mendelson. Continuing Education in Anaesthesia Critical Care \& Pain 2006;6:215-8.

4. Practice guidelines for preoperative fasting and the use of pharmacologic agents to reduce the risk of pulmonary aspiration: application to healthy patients undergoing elective procedures: an updated report by the American Society of Anesthesiologists Committee on Standards and Practice Parameters. Anesthesiology 2011:114:495-511.

5. Nygren J. The metabolic effects of fasting and surgery. Best Pract Res Clin Anaesthesiol 2006;20:429-38.

6. Practice guidelines for preoperative fasting and the use of pharmacologic agents to reduce the risk of pulmonary aspiration: Application to healthy patients undergoing elective procedures: An updated report by the american society of anesthesiologists task force on preoperative fasting and the use of pharmacologic agents to reduce the risk of pulmonary aspiration. Anesthesiology 2017;126:376-93. 